International Journal of Pure and Applied Mathematics

Volume $91 \quad$ No. 4 2014, 477-481

ISSN: 1311-8080 (printed version); ISSN: 1314-3395 (on-line version)

url: http://www.ijpam.eu

doi: http://dx.doi.org/10.12732/ijpam.v91i4.4

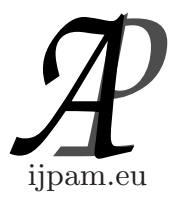

\title{
NEUTRAL OPERATORS AND TRIPLE REPRESENTATION
}

\author{
J. Vimala \\ Department of Mathematics \\ Alagappa University \\ Karaikudi, Tamilnadu, INDIA
}

\begin{abstract}
The basic idea of triple representation of a structure is to associate two simpler structures and a connecting map. Triple representation was first introduced by Chen and Gratzer to Stone Lattices [2], [3]. The concept was generalised to distributive pseudo-complemented lattices and then to distributive pseudo-complemented semilattices by Katrinak. The triple representation for modular Pseudo Complemented semilattice was suceeded by W.H.Cornish[4]. Now the triple representation was obtained for a supermodular semilattice and thereby the supermodular semilattices are characterised.
\end{abstract}

AMS Subject Classification: 06, 06B

Key Words: semilattice, modularlattice, supermodular, closure operator

\section{Introduction}

The congruence classes generated by neutral filters play a vital role in the triple representation of supermodular semilattices. In this section triple representation for supermodular semilattice has been obtained and some basic theorems which characterise supermodular semilattices are presented.

Definition 1.1. Let $S$ be a semilattice with the greatest element 1. A map $\pi: S \rightarrow S$ is called a closure operator if

1. $(x \pi) \pi=x \pi$

Received: November 20, 2013

(c) 2014 Academic Publications, Ltd. url: www.acadpubl.eu 
2. $x \leq y \Rightarrow x \pi \leq y \pi$

3. $x \leq x \pi$; hold for all $x, y \in S$.

Definition 1.2. Let $\pi$ be a closure operator on a semilattice $S$. Then

1. $C_{\pi}(S)=\{x \in S: x=x \pi\}$ is the set of $\pi$ - closed elements of $S$

2. $D_{\pi}(S)=\{x \in S: x \pi=1\}$ is the set of $\pi$ - dense elements of $S$.

3. Closure operator $\pi$ is called multiplicative if $(x \wedge y) \pi=x \pi \wedge y \pi$ for all $x, y$ in $S$.

4. A $p$ - closure operator $\pi$ on $S$ is a multiplicative closure operator if for each $s \in S$, there exist $c \in C_{\Pi}(S)$ and $d \in D_{\pi}(S)$ such that $s=c \wedge d$.It is also equivalent for each $s \in S$ there exists $d \in D_{\pi}(S)$ such that $s=s \pi \wedge d$

5. A multiplicative closure operator $\pi$ is called neutral if the filter $D_{\pi}(S)$, which is the set of $\pi$ - dense elements is a neutral filter.

Result 1.3. If the closure operator $\pi$ is multiplicative then

1. $C_{\pi}(S)$ is a subsemilattice of $S$

2. $D_{\pi}(S)$ is filter of $S$

Proposition 1.4. Let $\pi$ be a multiplicative closure operator on a semilattice $S$ with 1 . Then the map $\alpha: S / \phi\left(D_{\pi}(S)\right) \rightarrow C_{\pi}(S)$ is defined by $\left([s] \phi\left(D_{\pi}(S)\right)\right) \alpha=s \pi$ an isomorphism, if $\pi$ is a $p$-closure operator.

Conversely, if $\alpha$ is an isomorphism and $S$ is supermodular then $\pi$ is a $p$ closure operator.

Proof. Assume that $\pi$ is a $p$-closure operator on a semilattice $S$ with 1 . It can be easily verified that $\alpha: S / \phi\left(D_{\pi}(S)\right) \rightarrow C_{\pi}(S)$ defined by $\left([s] \phi\left(D_{\pi}(S)\right)\right) \alpha=$ $s \pi$ is an isomorphism,since $\pi$ is a $p$-closure operator.

Conversely, assume that $S$ is a supermodular semilattice with $1, \pi$ is a multiplicative closure operator and $\alpha$ is an isomorphism.

To prove that $\pi$ is a $p$ - closure operator. Let $s \in S$ be arbitrary.

$\Rightarrow[s] \phi\left(D_{\pi}(S)\right) \in S / \phi\left(D_{\pi}(S)\right)$

$\Rightarrow[s] \phi\left(D_{\pi}(S)\right)=[s \pi] \phi\left(D_{\pi}(S)\right)$, since $(s \pi) \pi=s \pi$

$\Rightarrow s \wedge d=s \pi \wedge d$ for some $d \in D_{\pi}(S) \Rightarrow s \pi \geq s \geq s \pi \wedge d$

$\Rightarrow s=s \pi \wedge d_{1}$ for some $d_{1} \geq d$ so that $d_{1} \in D_{\pi}(S)$ since $S$ is supermodular.

Thus $\pi$ is a $p$-closure operator. 
Theorem 1.5. If $\pi$ is a p-closure operator on a supermodular semilattice $S$ then $D_{\pi}(S)$ and $C_{\pi}(S)$ are both supermodular.

Proof. Given $\pi$ is a $p$-closure operator on a supermodular semilattice $S$. First to prove that $D_{\pi}(S)$ is a supermodular. $D_{\pi}(S)$ is a filter of a supermodular semilattice $S . \Rightarrow D_{\Pi}(S)$ is supermodular.

Next to prove that $C_{\pi}(S)$ is supermodular. Now $D_{\pi}(S)$ is a filter on $S$. $\Rightarrow \phi\left(D_{\pi}(S)\right)$ is a semilattice congruence.

$\Rightarrow S / \phi\left(D_{\pi}(S)\right)$ is supermodular.

Also $\alpha: S / \phi D_{\Pi}(S) \Rightarrow C_{\Pi}(S)$ is an isomorphism.

$\Rightarrow$ The subsemilattice $C_{\Pi}(S)$ is also supermodular.

Definition 1.6. Let $S$ be a meet semilattice with 1 and $T$ be a join semilattice with 0 . Then $\psi: S \longrightarrow T$ is called a 1 - dual homomorphism if $1 \psi=0$ and $(x \wedge y) \psi=x \psi \vee y \psi$ for each $x$ and $y$ in $S$.

Definition 1.7. If $S$ is a supermodular semilattice with 1 possessing a $p$-closure operator $\pi$ then $\psi_{\pi}^{\mathrm{s}}: C_{\pi}(S) \rightarrow \mathscr{F}\left(D_{\pi}(S)\right)$ is defined by $C \psi_{\pi}^{\mathrm{s}}=$ $\left\{d \in D_{\Pi}(S): d \geq c\right\}$ for each $c \in C$

Result 1.8. Result: Let $S$ be a supermodular semilattice, possessing a $p$-closure operator $\pi$. The mapping $\psi_{\pi}^{\mathrm{s}}: C_{\Pi}(S) \rightarrow \mathscr{F}\left(D_{\pi}(S)\right)$ is a 1-dual homomorphism if and only if $\pi$ is a neutral $p$-closure operator.

Definition 1.9. A $l$-dual homomorphism $\psi_{\pi}^{\mathrm{S}}$ is called the structure homomorphism associated with $S$ and $\pi$.

Definition 1.10. $\left(C_{\pi}(S)\right), \psi_{\pi}^{\mathbf{s}},\left(D_{\pi}(S)\right)$ is called the triple associated with the supermodular semilattice $S$ and neutral $p$-closure operator $\pi$.

Definition 1.11. An isomorphism $\psi: S \longrightarrow T$, where $S$ and $T$ are two semilattices with closure operators $\pi$ and $\rho$ respectively is defined as a closure isomorphism if $(s \psi) \rho=(s \pi) \psi$ for each $s \in S$.

Theorem 1.12. (Uniqueness Theorem) A supermodular semilattice $S$ with 1 and a neutral p-closure operator $\pi$ is such that itself and the closure operator are determined up to a closure ismorphism by the triple $\left(C_{\pi}(S)\right), \psi_{\pi}^{\mathbf{s}},\left(D_{\pi}(S)\right)$

Proof. $\left(C_{\pi}(S)\right), \psi_{\pi}^{\mathrm{s}},\left(D_{\pi}(S)\right)$ is the given triple. To find $S$ and $\pi$ from this triple. Construct the set $S_{1}$ as follows:

$S_{1}=\left\{\left(c,[d] \Theta\left(c \psi_{\pi}^{\mathbf{s}}\right)\right): c \in\left(C_{\Pi}(S)\right), d \in D_{\pi}(S)\right\}$. We define the infimum in $S_{1}$, by $x \wedge y=\left(a \wedge b,[d \wedge e] \Theta(a \wedge b) \psi_{\pi}^{\mathbf{s}}\right)$, where $x=\left(a,[d] \Theta\left(a \psi_{\pi}^{\mathbf{s}}\right)\right), y=$ $\left(b,[e] \Theta\left(b \psi_{\pi}^{\mathbf{S}}\right)\right)$.

It can be easily verified that $S_{1}$ is a semilatice. Now define the mapping $\mu: S \longrightarrow S_{1}$ by $s \mu=\left(s \pi,[d] \Theta\left((s \pi) \psi_{\pi}^{\mathbf{S}}\right)\right)$, where $d \in\left(D_{\pi}(S)\right)$ is such that 
$s=s \pi \wedge d$.

$\mu$ is both well defined and one to one.

$\mu$ is onto, since $\pi$ is $p$-closure

$\Rightarrow \mu$ is an isomorphism.

Let $\pi_{1}: S_{1} \longrightarrow S_{1}$ be defined by $\left\{(x \mu) \pi_{1}=\left(a,[1] \Theta\left(a \psi_{\pi}^{\mathbf{S}}\right)\right)\right\}$, where $x \mu=$ $\left.\left(a,[d] \Theta\left(a, \psi_{\pi}^{\mathbf{S}}\right)\right)\right\}$

Next to prove that $\mu: S \longrightarrow S_{1}$ is closure isomorphic. That is to claim that $(x \pi) \mu=\left((x \mu) \pi_{1}\right.$ where $\mu$ is an isomorphism.

Now consider $(x \pi) \mu=\left((x \pi) \pi,[d] \Theta\left((x \pi) \pi \psi_{\pi}^{\mathrm{S}}\right)\right.$, such that $x \pi=x \pi \wedge d$, for all $x \in S$ where $\left.\left.d \in\left(D_{\pi}(S)\right)\right)=\left((x \pi),[d] \Theta(x \pi) \psi_{\pi}^{\mathrm{S}}\right)\right), \Longrightarrow d \geq x \pi$ for all $x \in S$ $\Rightarrow d=1$

Therefore

$$
\begin{aligned}
\left.\left.(x \pi) \mu=\left(\left((x \pi),[1] \Theta\left((x \pi) \psi_{\pi}^{\mathbf{s}}\right)\right), x \pi \in C_{\Pi}(S)\right)\right)\right) & \\
& \Rightarrow(x \pi) \mu\left(a,[1] \Theta\left(a \psi_{\pi}^{\mathbf{s}}\right)\right), a \in C_{\Pi}(S) .
\end{aligned}
$$

By the definition of $\pi_{1}(x \mu) \pi_{1}=\left(a,[1] \Theta\left(a \psi_{\pi}^{\mathrm{S}}\right), a \in C_{\pi}(S)\right.$.

Therefore $(x \pi) \mu=(x \mu) \pi_{1}$.

Thus $\mu: S \longrightarrow S_{1}$ is closure isomorphic.

Next, $\pi_{1}$ is a $p$ - closure neutral operator: $\psi_{\pi}^{\mathrm{S}}$ is a 1 dual homomorphism $\Rightarrow \psi_{\pi_{1}}^{\mathrm{S}_{1}}$ is also a 1 dual homomorphism $\Rightarrow \pi_{1}$ is a neutral operator.

Thus a semilattice $S$ with 1 and a neutral $p$-closure operator $\pi$ are determined upto a closure isomorphism.

Definition 1.13. $(C, \psi, D)$ is also called the triple if both $C$ and $D$ are supermodular semilattices with 1 and $\psi$ is a 1-dual homomorphism of mapping $C$ into $\mathscr{F}(D)$.

\section{References}

[1] G.Birkhoff, Lattice Theory, Amer. Math. Soc. Colloq. Publ.XXV Providance R.I, Third Edition, Third Printing (1979).

[2] C.C.Chen and G.Gratzer, Stone lattices I, Construction and Theorems, Canad. J.Math, 21 (1969), 884-894.

[3] C.C.Chen and G.Gratzer, Stone lattices II, Construction and Theorems, Canad. J.Math, 21 (1969), 895-903.

[4] W.H. Cornish, Pseudo-Complemented Modular Semilattices, 239 - 251. 
[5] W.H. Cornish, Characterisations of distributive and modular semilattices, Math. Japanica, 22 (1977), 159-174.

[6] S. Hashimoto, S. Kinugawa, On neutral elements in lattices, Proc, and Japan Acad, 39 (1963), 162-163.

[7] W. Iqbalunnisa, On neutral elements in a lattice, J. Indian. Math. Soc, 28 (1964), 25-31.

[8] W. Iqbalunnisa and R. Natarajan, Supermodular semilattices, J. Madras Univ. section B, 48, No. 1 (1985), 4853-69.

[9] W. Iqbalunnisa and B. Vasantha, Supermodular lattices, J. Madras Univ., Section B, 44 (1981), 58-80. 
